\title{
Zur Determination der zeitlichen Verteilung von Fortpflanzungsprozessen in Laborkulturen des Polychaeten Typosyllis prolifera
}

\author{
H.-D. Franke \\ Zoologisches Institut der Technischen Universität; Pockelsstraße 10a, \\ D-3300 Braunschweig, Bundesrepublik Deutschland
}

\begin{abstract}
Factors controlling the timing of reproduction in laboratory cultures of the polychaete Typosyllis prolifera. Typosyllis prolifera (Krohn) from Poreč (Yugoslavia) has been cultured for 12 successive generations. The life cycle of the species in the laboratory is described briefly. During their life individuals reproduce several times (up to 15) by stolonization which, under constant laboratory conditions ( $L D 16: 8,20^{\circ} \mathrm{C}$ ), is cyclic and takes place about every 30 days. Based on the investigations of Durchon (1959) and Wissocq (1966), experiments on extirpation and transplantation of the proventriculus have been carried out. The results suggest that an endocrine system anatomically connected to the proventriculus is important in the control of reproduction. Most likely, the endogenous reproductive cycle of an individual is controlled by periodical changes of the activity of this system. During the period following stolonization, the endocrine system of the proventriculus, which at this time shows its maximal activity, inhibits sexual development and enables regeneration of the segments lost as stolon. A subsequent decrease of the hormonal activity induces sexual maturation and epitokous metamorphosis, thus leading to further stolonization. Exogenous factors influencing the timing of reproduction probably affect the endocrine function of the proventriculus. Short-day photoperiods (LD 10:14) and low temperatures $\left(12^{\circ} \mathrm{C}\right)$ given simultaneously (i.e. winter conditions) totally suppress reproduction. Under normally favourable conditions ( $\mathrm{LD} 16: 8,20^{\circ} \mathrm{C}$ ), reproductive processes can be prevented by starving or amputation of caudal segments. In all these cases, however, stolonization can be induced by removing the proventriculus. Exogenous factors also play a decisive role in synchronizing reproductive events within the species population. Under field conditions reproduction shows a lunar periodicity. The endogenous reproductive cycles of cultured specimens can be synchronized by a periodical light regime simulating the change of the moon.
\end{abstract}

\section{EINLEITUNG}

Die Steuerung der Fortpflanzungsprozesse und speziell die Bedeutung endokriner Mechanismen für die Kontrolle der Gametogenese und epitoken Metamorphose waren Gegenstand zahlreicher Untersuchungen an Polychaeten in den letzten 30 Jahren. Neuere zusammenfassende Darstellungen geben Durchon (1970), Golding (1972), Hauenschild (1974, 1975), Schroeder \& Hermans (1975) sowie Olive \& Clark (1978).

Die Familie Syllidae ist für ihre vielfältigen Fortpflanzungserscheinungen bekannt (Malaquin, 1893; Potts, 1911). Ein weit verbreiteter, die-Unterfamilien Syllinae und Autolytinae kennzeichnender Fortpflanzungsmodus ist die Stolonisation: Die atoken, benthischen Individuen bringen im Zuge eines der sexuellen Reifung parallel laufenden 
Metamorphoseprozesses epitoke Teilstücke (Stolonen) hervor, die nach Ablösung von ihrem Stammtier (Amme) ein kurzes, pelagisches Eigenleben führen und die Verbreitung sowie Abgabe der herangereiften Gameten übernehmen. Nach Erfüllung ihrer Aufgabe als Sexualstadien gehen die zu selbständiger Nahrungsaufnahme unfähigen Stolonen zugrunde; die am Boden zurückbleibenden Stammindividuen hingegen überleben den Stolonisationsprozeß, können die ihnen in Form der Stolonen verlorengegangenen Segmente regenerieren und erneut stolonisieren. Infolge Ausbildung eines Kopfes erlangen die Stolonen der Sylliden der Status von Individuen (Geschlechtstiere), ihr Bildungsprozeß erhält den Charakter einer ungeschlechtlichen Fortpflanzung, und der Entwicklungszyklus wird zur Metagenese.

Eine experimentelle Analyse der Stolonisation befindet sich noch im Anfangsstadium. Untersuchungen von Abeloos (1950), Durchon $(1950,1952)$ und Junqua (1957) an Typosyllis prolifera (als Syllis prolifera), Syllis amica und Trypanosyllis zebra erbrachten erste Hinweise auf die Existenz einer inhibitorischen Kontrolle der sexuellen Reifung und stolonialen Metamorphose: Die Amputation des vorderen, Kopf und Pharynxregion umfassenden Körperteils induziert im verbleibenden Wurmfragment eine Gametogenese und fortgesetzte Stolonisation. Eine Resektion allein des Prostomiums vermochte diesen Effekt nicht hervorzurufen. In selektiven Exstirpationsversuchen an verschiedenen Syllinen-Arten erwies sich der Proventrikel, ein charakteristischer Pharynxabschnitt der Sylliden (Abb. 1), als das Zentrum, unter dessen Einfluß die Fortpflanzung außerhalb der artspezifischen Fortpflanzungssaison (Syllis amica) bzw. unter Hungerbedingungen (Trypanosyllis zebra) verhindert wird (Durchon, 1957, 1959). An Syllis amica gelang Wissocq (1966) der Nachweis des hormonalen Charakters der vom Proventrikel über die Stolonisation ausgeübten inhibitorischen Kontrolle.

Eine Hemmung der geschlechtlichen Entwicklung durch den Vorderkörper ist auch bei den daraufhin untersuchten Autolytinen nachgewiesen. Im Unterschied zu den Syllinen kann eine Amputation des Vorderkörpers aber nicht in jedem Fall, und auch höchstens eine einmalige Stolonisation herbeiführen. Eine selektive ProventrikelExstirpation blieb stets unwirksam. Für Proceraea picta (als Autolytus pictus) wurde das Hemmzentrum ganz allgemein im Pharynxbereich (Durchon \& Wissocq, 1964), für Autolytus prolifer in der Proventrikelregion sowie den ihr unmittelbar folgenden Segmenten lokalisiert (Hauenschild, 1959; Schiedges, 1977).

Die geschlechtliche Differenzierung der Syllinen erwies sich wiederholt als labil: Die Stammtiere vermögen während aufeinanderfolgender Fortpflanzungsperioden Stolonen wechselnden Geschlechts hervorzubringen, wohingegen die Stolonen selbst getrenntgeschlechtlich sind (Durchon, 1951, 1959, 1975; Junqua, 1957; Wissocq, 1963-1964).

Alle früheren Untersuchungen an Syllinen erfolgten mit Freilandmaterial, dessen individuelle Vorgeschichte (Alter; Anzahl und Geschlecht bereits gebildeter Stolonen; Termin der letzten Fortpflanzung) unbekannt war und das nur für begrenzte Zeit ohne Ernährung im Labor am Leben erhalten wurde. Voraussetzung für eine weiterführende Analyse der Fortpflanzungsprozesse war die kontinuierliche Laborzucht eines geeigneten Untersuchungsobjektes. Die experimentellen Untersuchungen galten zwei Fragenkomplexen: der Determination des Fortpflanzungstermins durch endogene und exogene Faktoren sowie der Steuerung der sexuellen Differenzierung. Eine Kurzmitteilung über erste Ergebnisse liegt bereits vor (Franke, 1977). Die Determination des Fortpflanzungs- 
termins kann unabhängig vom Problem der sexuellen Differenzierung behandelt werden und ist Gegenstand der vorliegenden Arbeit. Alle die Steuerung der sexuellen Differenzierung betreffenden Fragen bleiben im folgenden unberücksichtigt und sind einer weiteren Veröffentlichung vorbehalten (Franke, in Vorbereitung).

\section{MATERIAL UND METHODEN}

\section{Untersuchungsobjekt}

Untersuchungsobjekt ist die kosmopolitische Sylline Typosyllis prolifera (Krohn, 1852), die seit mehr als 3 Jahren (12 Generationen) kontinuierlich im Labor gezüchtet wird. Das Ausgangsmaterial der Zucht wurde im April 1976 bei Poreč (Istrien) gesammelt. Typosyllis prolifera besiedelt das Phytal der obersten sublitoralen Zone und kann aus verschiedenem Algenmaterial, z. B. solchem der Gattung Halopteris, isoliert werden.

Die Fortpflanzung vollzieht sich an der istrischen Küste während einer ausgedehnten Fortpflanzungssaison. Zwischen April und September waren stets große Teile der untersuchten Population mit Prozessen der Stolonisation befaßt oder wiesen Anzeichen einer erst vor kürzerer Zeit stattgefundenen Teilung auf. Während der Wintermonate sind Fortpflanzungsprozesse vermutlich unterbunden: Im Frühjahr angetroffene Exemplare waren im Mittel deutlich größer als zu anderen Jahreszeiten, hatten also offensichtlich eine längere, von keiner Stolonisation unterbrochene Wachstumsperiode durchlaufen. Wie noch erläutert wird, lassen sich Fortpflanzungsvorgänge von Labortieren zuverlässig verhindern, wenn diese dem EinfluB von Kurztagbedingungen (LD $10: 14)$ und geringen Wassertemperaturen $\left(12^{\circ} \mathrm{C}\right)$ ausgesetzt werden.

\section{Kulturmethode}

Die Laborhaltung der Versuchstiere erfolgt in Form von Massen- und Einzelkulturen. Massenkulturen umfassen je etwa 50 Würmer, die gemeinsam in Polystyrolboxen $(10 \times 10 \times 6 \mathrm{~cm})$ mit $150 \mathrm{ml}$ belüftetem Kulturmedium gehalten werden; für die Einzelkultur finden kleine Glastiegel $(\varnothing 4 \mathrm{~cm})$ mit 2,5 ml Kulturmedium Verwendung, die in verschlossenen Polystyrolgefäßen untergebracht sind.

Die Kulturen befanden sich, sofern nicht anders vermerkt, in einem Raum mit 16stündigem Langtag (Leuchtstoffröhren vom Typ Tageslicht; Lichtintensität am Standort der Kulturen 2000-5000 Lux) und konstanter Temperatur von $20 \pm 1{ }^{\circ} \mathrm{C}$.

Als Kulturmedium dient künstliches, vor Gebrauch bei $80^{\circ} \mathrm{C}$ sterilisiertes Seewasser ("Tropic Marin Neu") der Dichte 1,025. Das Medium der Massenkulturen wird einmal, das der Einzelkulturen zweimal wöchentlich erneuert, wobei der Bodensatz in den Zuchtgefäßen verbleibt. Massenkultur-Tiere werden zudem im Abstand von 12, Einzelkultur-Tiere im Abstand von 3 Wochen in saubere Gefäße umgesetzt.

Der Boden der Kulturgefäße ist mit synthetischer Filterwatte ausgelegt, die erst dann entfernt werden darf, wenn sich ein umfangreicher, von den Würmern zerwühlter Bodensatz gebildet hat. Ohne eine solche Möglichkeit zu intensivem Kontakt mit Strukturen ihrer Umgebung verfallen die Tiere bald in Inaktivität oder umschlingen sich unter gegenseitiger Beschädigung. 
Als Futter wird den Kulturen jeweils nach dem Wechsel des Mediums eine Suspension der einzelligen, rasenbildenden Grünalge Platymonas zugesetzt. Außerdem erfolgt eine einmalige Impfung mit dem heterotrophen Dinoflagellaten Oxyrrhis, der ebenso wie Platymonas im Labor gezüchtet wird. Oxyrrhis ist ein wesentlicher Nahrungsfaktor für Jungtiere vor der Metamorphose des Pharynxapparates; ältere Stadien lassen sich hingegen ganz auf der Basis von Platymonas und gelegentlichen Zugaben fein zerteilten handelsüblichen Fischfutters ("TetraMin") ernähren. Ein effektiver Aufschluß der Platymonas-Zellen ist erst nach mehrmaliger Passage des Darmtrakts möglich; dieser Umstand bereitet gewisse Schwierigkeiten bei der Einzelhaltung von Tieren, die sich aber dadurch beheben lassen, daß nicht frisches, sondern angedautes Material aus Massenkulturen für die Ernährung Verwendung findet.

Um Nachzuchten zu erhalten, werden abgeschnürte Stolonen sowie unmittelbar vor der Teilung stehende Tiere den Massenkulturen entnommen und in Boverischalen zusammengebracht. Die geschlüpften Jungtiere werden auf Petrischalen $(\varnothing 10 \mathrm{~cm}$; Wasserstand $0,5 \mathrm{~cm}$ ) verteilt und mit Oxyrrhis sowie Platymonas ernährt. Hier verbleiben sie 6-10 Wochen (ein Wasserwechsel findet während dieser Zeit nicht statt) und werden nach Abschluß der Metamorphose in die beschriebenen Massen- bzw. Einzelkulturen überführt.

\section{Bemerkungen zum Entwicklungszyklus von Typosyllis prolifera in der Laborkultur}

Die reifen Gameten werden von den Stolonen während eines Schwärmvorganges zur Besamung ins Medium entlassen. Die Eier $(\varnothing 70 \mu \mathrm{m})$, von denen je nach Umständen 100 bis mehrere tausend pro $\&$ Stolon gebildet werden, sinken zu Boden, wo sich die kurze, maximal 48 Std. (bei $20^{\circ} \mathrm{C}$ ) beanspruchende Embryonalentwicklung vollzieht. Schlüpfstadium ist eine Metatrochophora, die unter Umgehung einer pelagischen Phase unmittelbar das Bodenleben aufnimmt. Bereits im Alter von 2 Tagen besitzen die Larven, deren geringe Dottervorräte nun erschöpft sind, einen funktionsfähigen Pharynxapparat und beginnen mit der Nahrungsaufnahme und dem teloblastischen Wachstum. Mit dem Auftreten der ersten beborsteten Parapodien ist am 6. Tag das Stadium der Borstenlarve erreicht.

Nach etwa 3 Wochen, in denen die Larven auf einen Umfang von 6-7 PpS (= parapodientragende Segmente) herangewachsen sind, beginnt die Metamorphose des Pharynxapparates. Hierbei erfährt das einfache, larvale Organ die für juvenile und adulte Stadien aller Sylliden charakteristische Gliederung in ein starres Schlund (Pharynx-)rohr und den muskulösen Proventrikel (Abb. 1). Der Prozeß der Pharynxmetamorphose geht mit einer deutlichen Verringerung der Wachstumsgeschwindigkeit einher und stellt eine kritische, verlustreiche Periode für die Aufzucht dar: Etwa zwei Drittel der Individuen eines Zuchtansatzes erweisen sich jeweils als unfähig, diesen Entwicklungsschritt unter den gegebenen Kulturbedingungen zu vollziehen, und gehen zugrunde.

Die Ausbildung des definitiven Pharynxapparates im Alter von etwa 6 Wochen (Körperumfang 9-12 PpS) markiert das Ende der Larvalentwicklung. Im Verlauf der sich anschließenden Juvenilentwicklung bildet die Proliferationszone etwa alle 2 Tage ein neues PpS, wobei jedoch erhebliche individuelle Unterschiede in der Wachstumsge- 


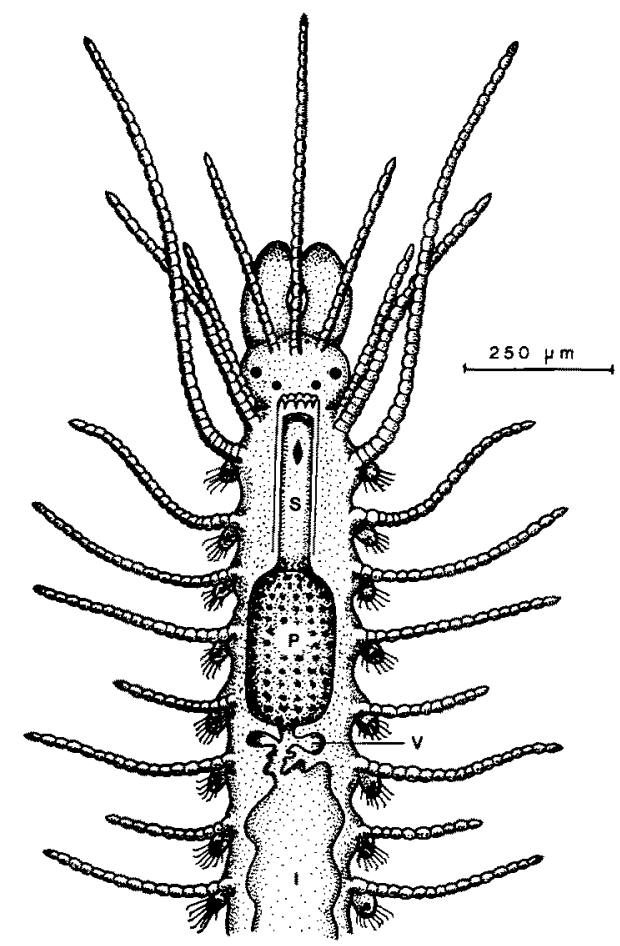

Abb. 1. Vorderkörper einer juvenilen Typosyllis prolifera (Alter 8 Wochen, Körperlänge 3,5 mm, 18 $\mathrm{PpS}=$ parapodientragende Segmente) mit den Differenzierungen des Darmtraktes. I: Intestinum;

P: Proventrikel mit Drüsenringen; S: Schlund- (Pharynx-)Rohr mit Schlundzahn; V: Ventrikel

schwindigkeit auftreten. Der für den Eintritt in die erste Fortpflanzungsperiode erforderliche Körperumfang von 35-40 PpS (Körperlänge 8-12 mm) wird von besonders schnellwüchsigen Exemplaren bereits im Alter von 10 Wochen erreicht, während manche Tiere hierfür mehr als den doppelten Zeitraum benötigen. Das mittlere Alter beim erstmaligen Eintritt in eine Stolonisationsphase beträgt 13,5 Wochen. Im Mittel etwa $15 \%$ der geschlüpften Tiere eines Zuchtansatzes erlangen unter den beschriebenen Kulturbedingungen die Geschlechtsreife.

Der Stolonisationsvorgang umfaßt in zeitlich-räumlicher Koordination die Prozesse der sexuellen Reifung, stolonialen Metamorphose und Regeneration des Stammtieres. Die Stolonen von Typosyllis prolifera entstehen in Einzahl als Ergebnis einer heteromorphen paratomen Querteilung.

Erstes Zeichen eines beginnenden Stolonisationsprozesses, der 13-15 Tage später mit der Abschnürung eines Stolons seinen AbschluB findet, ist das Erscheinen von Gametogenese-Stadien im caudalen Körperabschnitt des Stammindividuums. Die zunächst hyalinen Oocyten werden deutlich als solche von außen erkennbar, sobald sie einen $\varnothing$ von 15-20 $\mu \mathrm{m}$ erreicht haben; eine milchige Spermatogonienmasse sowie die auffällige Hypertrophie von Drüsen der Segmentalorgane kennzeichnen eine einsetzende $\delta$ Differenzierung. Die Cölome der anschwellenden Geschlechtssegmente werden vollständig von den heranreifenden Gameten erfüllt. Der sehr weite Darmtrakt 
erfährt in der Geschlechtsregion eine Reduktion zu einem englumigen Kanal, bleibt aber weiterhin durchgängig; die gesamte Periode der geschlechtlichen Entwicklung ist von intensiver Nahrungsaufnahme begleitet.

8-9 Tage nach dem äußerlich erkennbaren Eintritt in eine Fortpflanzungsperiode bilden sich an einem der vorderen Geschlechtssegmente 2 Paar rote Augenflecken, markieren das prospektive stoloniale Kopfmetamer und geben damit die Lage der künftigen Teilungsebene zwischen Stammtier und Stolon zu erkennen. Der Teil der Geschlechtsprodukte, der außerhalb der prospektiven Stolonregion heranreift, wird unmittelbar vor dem Teilungsvorgang in den reifen Stolon verlagert, der auf diese Weise die Gesamtheit der gebildeten Gameten zugeteilt bekommt.

Die caudale Segmentproliferation läuft auch während der sexuellen Reifung zunächst mit fast unverminderter Intensität weiter und wird erst im Anschluß an das Erscheinen der stolonialen Augenflecken eingestellt. Zugleich beginnt bereits auf diesem Stadium, d. h. etwa 1 Woche vor Ablösung des Stolons, die Regeneration des Stammtieres, deren eigentümlicher Verlauf wiederholt beschrieben worden ist $(z)$.$B .$ Okada, 1929). Zum Zeitpunkt der Teilung trägt das zurückbleibende Stammindividuum folglich bereits ein caudales Regenerat, das ein Pygidium und 2-6 Segmente mit Parapodienanlagen umfaßt.

Parallel zur sexuellen Reifung und einsetzenden Stammtier-Regeneration unterziehen sich die in die Stolonbildung eingehenden Segmente der epitoken Metamorphose, die zu der ausgeprägten Heteromorphie zwischen benthischem Stammtier und pelagischem Sexualstadium führt. Die bedeutendsten Umbildungsvorgänge sind: die Differenzierung des prospektiven ersten Stolonsegments zum typisch gestalteten Stolonkopf mit extrem großen Augen und besonderen Kopfanhängen; die Metamorphose der Parapodien unter Ausbildung spezieller Kapillarborsten-Bündel; die Umbildung der Segmentalorgane zu Gonodukten, die im $\delta$ Geschlecht auffällige Anhangsdrüsen tragen; die bereits erwähnte Reduktion des Darmtrakts zu einem englumigen, aber weiterhin durchgängigen Kanal; die grundlegende Umorganisation des Muskelsystems, welche die schnellen Schwimmbewegungen schwärmender Stolonen ermöglicht.

Die Stolonen sind getrenntgeschlechtlich; ein Sexualdimorphismus, wie er die Stolonen der Autolytinen auszeichnet, fehlt den Syllinen. Auch der zeitliche Verlauf von geschlechtlicher Reifung und stolonialer Metamorphose läßt keine geschlechtsspezifischen Unterschiede erkennen.

13-15 Tage nach dem äußerlich erkennbaren Eintritt in eine Fortpflanzungsperiode vollzieht sich die Trennung des Stolons von seinem Stammtier. Alle Teilvorgänge der sexuellen Reifung und epitoken Metamorphose sind zu diesem Zeitpunkt abgeschlossen und die Stolonen somit unmittelbar bei Aufnahme ihres Eigenlebens selbst fortpflanzungsbereit. Die Lebensdauer dieser mund- und pharynxlosen Geschlechtstiere ist auf wenige Tage beschränkt, innerhalb derer sie ihre Aufgabe als Sexualstadien, nämlich die Verbreitung und koordinierte Abgabe der Gameten, zu erfüllen haben.

Im AnschluB an den TeilungsprozeB wird die schon vorher begonnene Regeneration des Stammindividuums intensiv fortgeführt. Unter den herrschenden Kulturbedingungen $\left(20^{\circ} \mathrm{C}\right.$, LD 16:8, reichliche Ernährung) können die dem Stammtier durch die Stolonisation verlorengegangenen Segmente innerhalb von 2-3 Wochen ersetzt und die Voraussetzungen für eine erneute Stolonisation geschaffen werden. Das Fortpflanzungsgeschehen folgt einer ausgeprägten Rhythmik (siehe unten): Gut ernährte Labortiere 
kommen im Wechsel mit der Regeneration im Mittel etwa alle 30 Tage erneut zur Fortpllanzung.

Bei den wiederholten Stolonisationen eines Individuums ist die Lage der Teilungsebene nicht konstant. Zunächst übertrifft die Anzahl der zwischen zwei Fortpflanzungsvorgängen neugebildeten $\mathrm{PpS}$ diejenige, die in die Bildung des jeweils neuen Stolons eingeht. Die Labortiere, die bei einem Umfang von etwa 35-40 PpS in ihre erste Fortpflanzungsperiode eintreten, wachsen folglich trotz der in mehr oder weniger regelmäßigem Abstand von etwa 30 Tagen wiederholten Stolonisation weiter heran, bis sie nach 4-6 Fortpflanzungszyklen ihren Endumfang von 60-70 PpS (Körperlänge 18-22 $\mathrm{mm}$ ) erreicht haben. Von nun an bleibt der mittlere Segmentumfang eines Individuums über die weiteren Teilungsvorgänge hinweg etwa konstant: Die Anzahl der im Anschluß an eine Teilung neugebildeten PpS entspricht im Mittel etwa derjenigen, die in Form des Stolons auch wieder verlorengeht.

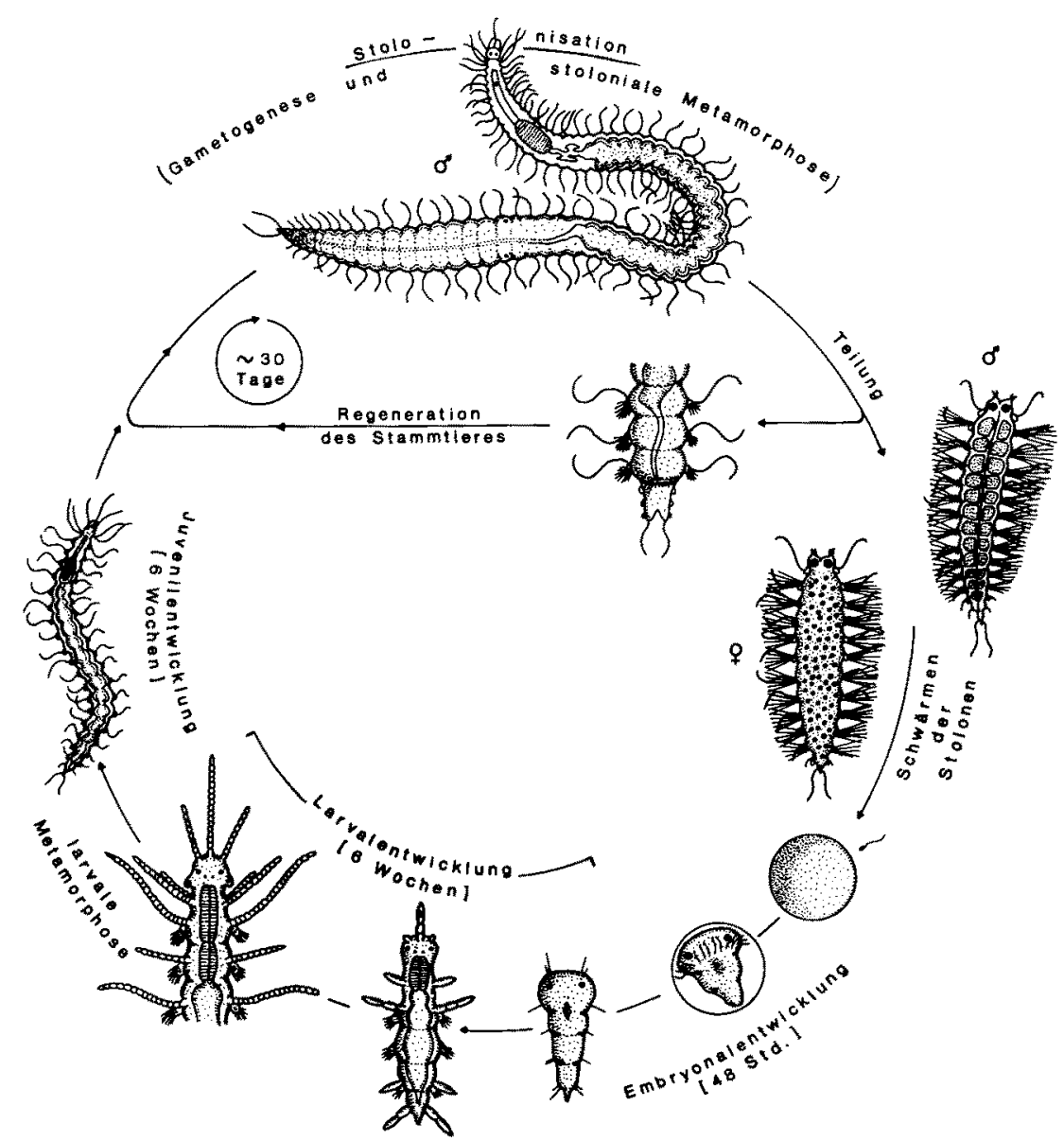

Abb. 2. Lebenszyklus von Typosyllis prolifera in der Laborkultur. Die verschiedenen Stadien sind nicht in den natürlichen Größenverhältnissen dargestellt 
Mehr als 600 Tiere wurden von der 10. Woche an für die Dauer ihres gesamten übrigen Lebens in Einzelkultur beobachtet: Die mittlere Lebensdauer der Würmer, die das Adultstadium erlangten (598 Exemplare), betrug 322 Tage, die mittlere Anzahl der durchgeführten Teilungen 7,3. Das langlebigste Exemplar erreichte ein Alter von 547 Tagen und hatte sich $15 \mathrm{mal}$ durch Stolonisation fortgepflanzt.

Abbildung 2 gibt eine zusammenfassende Darstellung der wichtigsten Stadien und Entwicklungsperioden im Lebenszyklus von Typosyllis prolifera.

\section{Operationstechnik}

Alle Experimente erfolgten mit gezüchtetem Tiermaterial. Sofern nicht anders vermerkt, waren die Versuchstiere unter konstanten Bedingungen in Massenkulturen aufgezogen worden, hatten bei Versuchsbeginn ein Alter von 28-32 Wochen erreicht und bereits 4-6 Fortpflanzungsprozesse vollzogen.

Nach 5-10minütiger Narkose in einer dem Kulturmedium isotonischen $\mathrm{MgCl}_{2}$ Lösung kamen die Versuchstiere zur Operation auf einen Objektträger, der mit einer etwa $1 \mathrm{~mm}$ starken Schicht eines hochviskosen Methylcellulose-Gels überzogen war. Die mikrochirurgischen Manipulationen wurden freihändig unter einem Stereomikroskop bei 40facher Vergrößerung ausgeführt.

Proventrikel-Exstirpationen: Mit einem speziellen Mikroskapell wurden die Würmer in der Proventrikelregion durch einen dorsolateralen Längsschnitt (Länge etwa 1,5 $\mathrm{mm}$ ) eröffnet. Der Proventrikel konnte daraufhin mit einer hakenförmigen Präpariernadel hervorgezogen und mittels einer Mikroschere aus dem Darmverband herausgeschnitten werden. Drei feine Haarschlingen, die bereits vor Beginn der Operation den betäubten Tieren umgelegt worden waren, wurden nach der Proventrikel-Entnahme in den Bereich der Operationswunde verschoben und zur Herbeiführung eines Wundverschlusses festgezogen.

Proventrikel-Implantationen: Eine Implantation von Proventrikeln in das Cölom intakter Würmer ist aus räumlichen Gründen unmöglich, das fast das gesamte Körperlumen der sehr schlanken Tiere von ihrem weiten Darmtrakt erfüllt wird. Implantationen erfolgten daher entweder als Reimplantation entnommener Organe zurück an ihre ehemalige Position oder in Form einer Transplantation an den Ort zuvor exstirpierter empfängereigener Proventrikel. Das einem Spendertier entnommene Organ wurde dem Empfänger mit Hilfe feiner Präpariernadeln durch die bei der vorausgegangenen Entnahme seines eigenen Proventrikels verursachte Operationswunde hindurch implantiert. Wie im Fall der Exstirpationen erfolgte anschließend ein WundverschluB mittels dreier Schnürungen, die zugleich eine mögliche Rejektion des Implantats verhinderten. Zur Erleichterung des Implantationsvorganges wurden den Empfängertieren jeweils Spender von etwas geringerer Körpergröße (aber vergleichbaren Alters) zugeordnet.

Exstirpationen und Implantationen von Proventrikeln wurden stets einschließlich der mit diesem Organ verwachsenen Bindegewebshüllen durchgeführt, die Elemente des stomatogastrischen Nervensystems enthalten.

Die ersten 3 Tage nach einer Operation verbrachten die Versuchstiere in täglich mehrmals gewechseltem, sterilem Seewasser; später erfolgte ein Wasserwechsel nur noch zweimal wöchentlich. Auf antibiotische Zusätze zum Medium konnte verzichtet 
werden. Operierte Tiere wurden unter denselben Bedingungen (LD 16:8, $20^{\circ} \mathrm{C}$ ) gehalten, denen auch die Zuchten ausgesetzt waren; obgleich sie zu weiterer Nahrungsaufnahme unfähig sind, können sie die Operation unter Umständen um mehr als 3 Monate überleben. Tiere, die einen operativen Eingriff nicht um mindestens 45 Tage überlebten, wurden als Operationsverluste gewertet, es sei denn, sie waren bereits vorher zur Fortpflanzung gelangt. Die Verluste betrugen bei Exstirpationen etwa $10 \%$, bei Transplantationen fast $40 \%$. Die Kontrolle der postoperativen geschlechtlichen Entwicklung erfolgte unter einem Stereomikroskop bei 100facher Vergrößerung in gemischtem Durch- und Auflicht.

\section{ERGEBNISSE}

\section{Der endogene Fortpflanzungsrhythmus}

In Massenkulturen erfolgen Fortpflanzungsprozesse gleichmäßig über die Zeit verteilt. Dieser Gleichverteilung liegen ausgeprägte, unter den vorliegenden konstanten Bedingungen jedoch unsynchronisierte individuelle Rhythmen zugrunde. 310 Tiere von gutem Allgemeinzustand (gefüllter Darm, gut entwickelte Cirren) wurden an einem Tag, in dessen Morgenstunden sie sich unter Abschnürung eines Stolons fortgepflanzt hatten, zur Beobachtung der zeitlichen Verteilung weiterer Fortpflanzungsereignisse aus Massen- in Einzelkulturen überführt und über einen Zeitraum von 4 Monaten täglich kontrolliert. Das Ergebnis zeigt Abbildung 3.

Mit Ausnahme von 15 Individuen, die vorher zugrunde gingen oder aus anderen Gründen nicht mehr stolonisierten, kamen alle Tiere zwischen dem 26. und 39. Tag, im

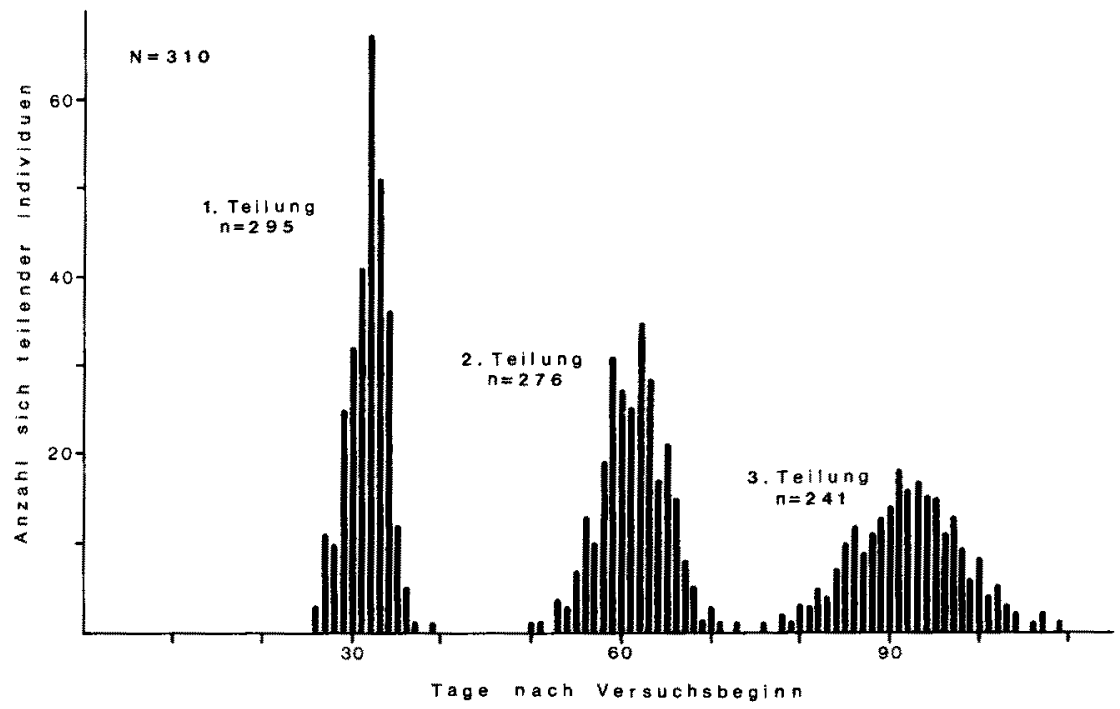

Abb. 3. Zeitliche Verteilung von Fortpflanzungsprozessen intakter, ernährter Einzelkultur-Tiere unter konstanten Bedingungen $\left(20^{\circ} \mathrm{C}\right.$, LD 16:8) bezogen auf den Tag der letzten Teilung in Massenkulturen (= Versuchsbeginn) 
Mittel 31,7 Tage nach der letzten, noch in Massenkulturen vollzogenen Teilung erneut zur Fortpflanzung. Erste Anzeichen für eine neue Stolonisationsperiode zeigten sich im Mittel um den 17. Tag herum, frühestens am 11., spätestens am 23. Tag. Die mittlere Anzahl der zwischen den Teilungen neugebildeten PpS betrug 15,8 und entsprach damit etwa dem mittleren PpS-Umfang der Stolonen $(14,6)$. Versuchstiere, die diese erste Fortpflanzung in Einzelkulturen hinreichend lange überlebten (276 Exemplare), teilten sich ein weiteres Mal zwischen dem 50. und 73. Tag, im Mittel 61,3 Tage nach Versuchsbeginn bzw. 29,6 Tage nach der vorausgegangenen Stolonisation. Die mittlere Anzahl der zwischenzeitlich neugebildeten PpS betrug 14,9, die mittlere Stolonengröße 14,8 PpS. Die 3. Stolonisation in Einzelkultur (241 Tiere, mittlere Stolonengröße 15,2 PpS) vollzog sich nach Neubildung von im Mittel 14,1 PpS zwischen dem 76, und 109. Tag, im Mittel 91,9 Tage nach Versuchsbeginn. Mit dem 98. Tag gelangten jedoch bereits die ersten Exemplare zur 4. Teilung; diese 4. Teilungsserie, die sich mit der 3. Serie überschneidet, ist in Abbildung 3 nicht mehr berücksichtigt.

Die Fortpflanzungsereignisse wiederholt stolonisierender Individuen folgen rhythmisch aufeinander: Gut ernährte Tiere bringen unter den gegebenen konstanten Bedingungen $\left(20^{\circ} \mathrm{C}\right.$, LD 16:8) etwa alle 30 Tage einen neuen Stolon zur Reife, und zwar unabhängig von dessen Geschlecht. Zwischen aufeinanderfolgenden Teilungen wurden jeweils etwa so viele Segmente neugebildet wie anschließend in Gestalt der Stolonen auch wieder verlorengingen. Die mittlere PpS-Anzahl der Vesuchstiere blieb daher während des Beobachtungszeitraumes trotz wiederholter Stolonisation etwa konstant. Die individuellen, unter konstanten Bedingungen unsynchronisierten Fortpflanzungsrhythmen sind offensichtlich endogener Natur und bedürfen auch keines AnstoBes durch eine exogene Periodik. Ihre mittlere spontane Periodenlänge von etwa 30 Tagen entspricht derjenigen (synodisch-)lunarer Periodizitätsphänomene.

\section{Die hormonale Steuerung des Fortpflanzungsrhythmus durch den Proventrikel}

Hinsichtlich der möglichen Bedeutung eines Proventrikel-Inkrets für die Kontrolle der zeitlichen Verteilung von Fortpflanzungsprozessen standen zwei Hypothesen zur Entscheidung: (1) Die endokrine Funktion des Proventrikels (vorausgesetzt, diese läßt sich am Versuchsobjekt nachweisen) ist auf die Verhinderung von Stolonisationsvorgängen außerhalb der Fortpflanzungssaison beschränkt. Unter dem Einfluß bestimmter Außenbedingungen (z. B. Photoperiode, Temperatur u. a.), wie sie im Labor hergestellt und konstantgehalten werden, entfällt diese Hemmung. Der dann auftretende rhythmische Wechsel von Wachstum (Regeneration) einerseits sowie sexueller Reifung und stolonialer Metamorphose andererseits ist ein sich selbst rückkoppelnd steuernder ProzeB und keiner weiteren endokrinen Kontrolle unterworfen. (2) Der Proventrikel ist in einer noch näher zu bestimmenden Weise als hormonales Zentrum an der Steuerung des Fortpflanzungsrhythmus beteiligt. Exstirpations- und Transplantationsexperimente haben diese Frage im Sinne der Hypothese 2 entscheiden können.

\section{Fortpflanzungsprozesse im Hungerzustand}

Von einer Proventrikel-Exstirpation (mit oder ohne nachfolgender Implantation) betroffene Versuchstiere sind zu weiterer Nahrungsaufnahme unfähig. Die Beurteilung der nach einem solchen Eingriff auftretenden Effekte erfordert somit, einen möglichen 

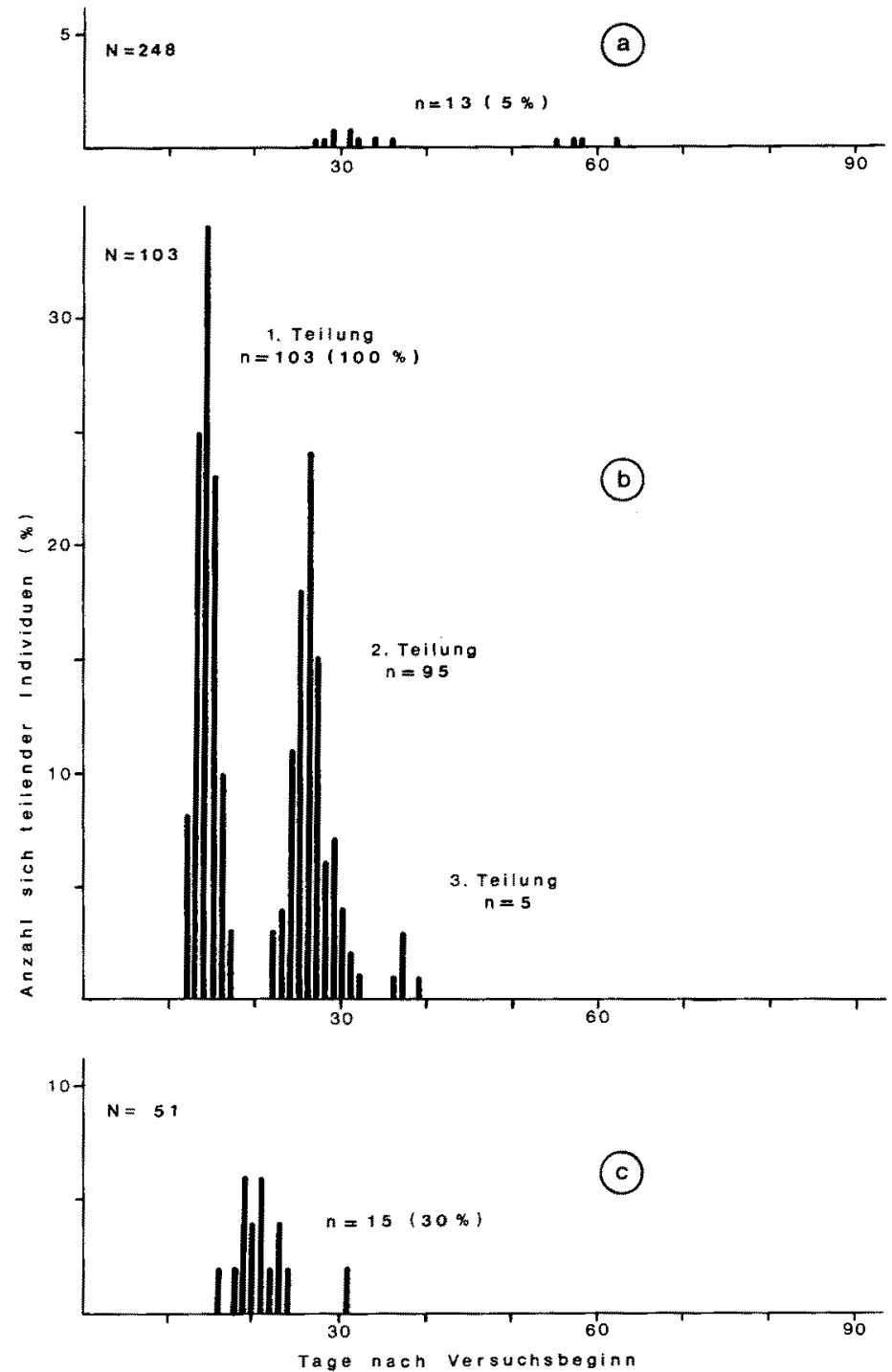

Abb. 4. Zeitliche Verteilung der Fortpflanzungsprozesse (Häufigkeiten in \% der Ausgangsanzahl N verwendeter Versuchstiere) von Hungertieren (a), Tieren nach Proventrikel-Exstirpation (b) und Tieren nach Proventrikel-Exstirpation mit direkt anschließender Reimplantation (c). Versuchsbeginn ist der Tag einer Teilung in Massenkultur

Einfluß von Hungerbedingungen auf die Fortpflanzungsvorgänge zu berücksichtigen. 248 Tiere wurden unmittelbar im Anschluß an eine in Massenkulturen vollzogene Teilung zur weiteren, täglichen Kontrolle in Einzelkulturen überführt und für einen Zeitraum von 4 Monaten totalem Nahrungsentzug ausgesetzt. Fast alle Tiere waren nach 60tägiger Hungerperiode noch am Leben, etwa die Hälfte (127) überstand sogar die gesamte Hungerzeit. 
Nur 13 Exemplare (etwa $5 \%$ ) gelangten während dieser Zeit zur Fortpflanzung, und auch stets nur ein einziges Mal (Abb. 4a). Die Teilung trat entweder zwischen dem 27. und 36. Tag oder zwischen dem 55. und 62. Tag ein, erfolgte also in zeitlicher Ubereinstimmung mit den Teilungsmaxima ernährter Tiere. Während der gesamten Hungerperiode wurden im Mittel nur 3,9 neue $\mathrm{PpS}$ gebildet, was ausschließlich innerhalb der ersten 20 Tage geschah. Diejenigen Tiere, die auch im Hungerzustand noch zur Fortpflanzung kamen, unterschieden sich in dieser Hinsicht nicht von der Masse der anderen Individuen. Die Bildung von Stolonen unter Hungerbedingungen vollzog sich somit fast ausschließlich auf Kosten älterer Segmente. Dieser Befund zeigt, daß der praktisch vollständige Ausfall von Fortpflanzungsprozessen im Hungerzustand primär nicht durch das Fehlen eines möglicherweise allein für die sexuelle Reifung und epitoke Metamorphose kompetenten Regenerats bedingt ist, sondern andere Ursachen hat.

\section{Fortpflanzungsprozesse nach selektiver Proventrikel-Exstirpation}

112 Tieren wurde an einem Tag, an dem sie sich durch Teilung fortgepflanzt hatten, selektiv der Proventrikel entfernt. Alle Versuchstiere befanden sich somit zum Zeitpunkt des an ihnen vorgenommenen operativen Eingriffs in der gleichen Phase des Fortpflanzungszyklus. 103 Individuen überlebten die Exstirpation: 53 Tiere, die bei Versuchsbeginn $\delta$ Stolonen, und 50 Tiere, die $q$ Stolonen hervorgebracht hatten. Bei allen postoperativen Fortpflanzungsprozessen wurden ausschließlich $\delta$ Stolonen zur Reife gebracht; Tiere, die zuvor noch $q$ Stolonen gebildet hatten, erfuhren somit eine quantitative und stabile Maskulinisierung. Dieser die sexuelle Differenzierung betreffende Befund bleibt im folgenden unberücksichtigt (vgl. "Einleitung"). Die zeitliche Verteilung von Fortpflanzungsprozessen nach selektiver Proventrikel-Exstirpation zeigt Abbildung 4b.

Alle Versuchstiere traten bereits kurze Zeit nach der Operation in eine neue Stolonisationsperiode ein; erste Anzeichen hierfür waren im Mittel um den 6. Tag herum zu erkennen. Die Teilung vollzog sich zwischen dem 12. und 17. Tag, im Mittel 14,1 Tage nach der Operation. Der mittlere zwischenzeitliche Segmentzuwachs betrug 3,7 $\mathrm{PpS}$, die mittlere Stolonengröße 20,5 PpS. Die Stolonenbildung erfolgte somit wie im Fall der (seltenen) Fortpflanzungsereignisse intakter Hungertiere fast ausschließlich auf Kosten älterer Segmente. Alle Tiere, die den 1. postoperativen Teilungsprozeß überlebten (95 von 103) traten unmittelbar anschließend in eine weitere Fortpflanzungsperiode ein; eine zwischenzeitliche Segmentneubildung unterblieb. Die Teilung erfolgte zwischen dem 22. und 32. Tag, im Mittel 26,2 Tage nach Versuchsbeginn bzw. 12,0 Tage nach der 1. Teilung. Die mittlere Stolonengröße betrug 15,5 PpS. Mit Abschluß dieser 2. postoperativen Stolonisation hatten die Versuchstiere in unmittelbarer Aufeinanderfolge ohne Unterbrechung durch Regenerationsperioden jeweils 3 Stolonen hervorgebracht, von denen die beiden unter experimentellen Bedingungen gebildeten Geschlechtstiere einen anormal großen relativen Segmentumfang aufwiesen. Nachdem auf diese Weise fast ihr gesamter postpharyngealer Körperabschnitt zu Stolonen umgewandelt und verlorengegangen war, starben die verbliebenen Stammtier-Fragmente innerhalb kurzer Zeit ab. Nur 5 von 95 Exemplaren besaßen noch eine hinreichend große Anzahl postpharyngeal gelegener Segmente, um eine weitere, sich wiederum direkt anschließende Stolonisation durchzuführen, bevor auch sie zugrunde gingen. 
Die Stolonisationen proventrikelloser Tiere verliefen in einer den Fortpflanzungsvorgängen intakter Individuen grundsätzlich vergleichbaren Weise. Abweichungen vom normalen Stolonisationsverlauf, wie sie z. T. auch von Durchon $(1950,1952)$ an Syllis amica beobachtet worden sind, zeigten sich im Ausbleiben der normalerweise etwa 1 Woche vor der Teilung einsetzenden Stammtier-Regeneration sowie hinsichtlich der Determination des Teilungsniveaus. In mehr als einem Drittel der Fälle kam es zu keiner eindeutigen Entscheidung über das künftige Teilungsniveau; stoloniale Kopfstrukturen entstanden nicht nur an einem, sondern an mehreren bis zahlreichen Segmenten (Polycephalie), deren Vordergrenzen sämtlich zu potentiellen Teilungsebenen wurden. Die relative Stolonengröße (=PpS-Anzahl des Stolons bezogen auf die PpSAnzahl des Gesamttieres), die unter Normalbedingungen im Mittel 0,23 betrug, war deutlich zugunsten der Stolonen verändert (im Mittel auf 0,39). Eine ProventrikelExstirpation beeinflußt den Determinationsmechanismus des Teilungsniveaus also sowohl in Richtung auf eine verminderte Eindeutigkeit als auch in quantitativer Hinsicht.

Durch eine Exstirpation des Proventrikels können Fortpflanzungsprozesse experimentell induziert werden, die bei ebenfalls hungernden, aber intakten Tieren fast vollständig unterbunden sind. Ein Proventrikelverlust versetzt die hiervon betroffenen Versuchstiere in einen permanenten Stolonisationszustand: Eine Serie unmittelbar, $d . h$. unter Fortfall der Regenerationsphasen, aufeinanderfolgender und zudem beschleunigt ablaufender Stolonisationen führt zur sukzessiven Umwandlung fast des gesamten Individuums in Geschlechtstiere und endet mit dem Tod des letztlich nur noch Kopf und Pharynxregion umfassenden Stammtier-Fragments.

Selbst nach einer lang ausgedehnten Hungerzeit vermag eine Proventrikel-Exstirpation Stolonisationsprozesse zuverlässig herbeizuführen: 25 Tieren, die eine 120tägige Hungerperiode ohne Fortpflanzung hinter sich hatten, wurde anschließend der Proventrikel entfernt. Alle überlebenden Individuen (21) gelangten daraufhin innerhalb von 18 Tagen zur Teilung. Infolge des durch die lange Hungerzeit bedingten ReservestoffMangels konnten jeweils nur geringe Mengen an Geschlechtsprodukten heranreifen. In 4 Fällen kam es sogar zur Bildung vollständig steriler, aber dennoch morphologisch normaler Stolonen. Dieser letztgenannte Befund demonstriert die auch von anderen epitoken Polychaeten bekannte prinzipielle Unabhängigkeit der Metamorphoseprozesse von der ihnen normalerweise vorangehenden Phase der geschlechtlichen Reifung. Weder das Fehlen eines caudalen Regenerats noch der auftretende Mangel an Nährstoffen selbst kann nach den obigen Ausführungen für den Ausfall von Fortpflanzungsprozessen unter Hungerbedingungen verantwortlich gemacht werden. Unmittelbare Ursache dieses Effektes ist vielmehr eine zentrale Hemmung, die durch ProventrikelExstirpation jederzeit aufgehoben werden kann.

Ein Vergleich der zeitlichen Verteilung von Fortpflanzungsprozessen proventrikelloser Tiere (Abb, $4 \mathrm{~b})$ mit derjenigen intakter, ernährter Individuen (Abb. 3) ergibt den ersten Hinweis auf eine Bedeutung des Proventrikels auch für die Steuerung des natürlichen Stolonisationsrhythmus. Tiere, denen direkt im Anschluß an eine Teilung der Proventrikel entfernt worden war, gelangten im Mittel 17,5 Tage früher zur nächsten Teilung als die im 30-Tage-Rhythmus stolonisierenden ernährten Individuen und hatten im Mittel bereits eine 2. Teilung vollzogen, bevor bei der Masse der letzteren der 1. Stolonisationsprozeß abgeschlossen war. Zwei verschiedene Umstände hatten hieran 


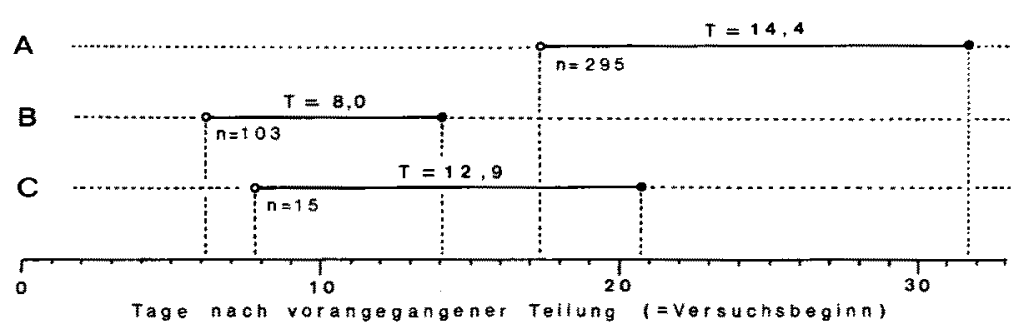

Abb. 5. Mittlerer Termin des äußerlich erkennbaren Eintritts in eine Stolonisationsperiode $(O)$ und mittlerer Teilungstermin ( $\bullet$ ) bezogen auf den Tag der vorausgegangenen Teilung (bei B und C zugleich Operationstermin), T: mittlere Dauer der Stolonisationsperiode (in Tagen); A: intakte, ernährte Tiere; B: Tiere nach Proventrikel-Exstirpation; C: Tiere nach Proventrikel-Exstirpation mit Reimplantation (sofern diese noch zur Fortpflanzung gelangten)

Anteil: die Vorverlegung des äußerlich erkennbaren Eintritts in eine neue Fortpflanzungsperiode um in Mittel 11,2 Tage auf Kosten eines fast vollständigen Ausfalls der Regenerationsperiode sowie der beschleunigte Ablauf des geschlechtlichen Reifungsprozesses (und zwar sowohl der Gametogenese als auch der Metamorphose), wie er in der Verkürzung des Zeitraumes zwischen dem sichtbaren Eintritt in eine Fortpflanzungsperiode und der eigentlichen Teilung von im Mittel 14,4 auf 8,0 Tage zum Ausdruck kommt (Abb. 5).

\section{Unterdrückung der Exstirpationseffekte durch Proventrikel-Reimplantation}

Die Ergebnisse der Exstirpationsexperimente geben begründete Hinweise auf eine Bedeutung des Proventrikels sowohl für die Hemmung der Fortpflanzungsprozesse unter Hungerbedingungen als auch für die Kontrolle des normalen Stolonisationsrhythmus. Es fehlt jedoch bisher der definitive Beweis dafür, daß die durch ProventrikelExstirpationen hervorgerufenen Effekte die spezifische Folge allein des Proventrikelverlustes und keine Reaktion auf eine anderweitige mit dem operativen Eingriff verbundene Veränderung am Versuchsobjekt darstellen. Darüber hinaus ist auch der Nachweis des mutmaßlich endokrinen Charakters der vom Proventrikel über die Fortpflanzungsvorgänge ausgeübten Kontrolle noch zu erbringen. Einer Gruppe von Versuchstieren (51 überlebende Exemplare: $27 q q$ und $\left.24 \delta^{\circ}\right)$ wurde zu diesem Zweck am Tage einer Teilung der Proventrikel entfernt, aber unmittelbar anschließend wieder implantiert. Die zeitliche Verteilung postoperativer Fortpflanzungsprozesse zeigt Abbildung 4c. Die Angabe aller Häufigkeiten in Prozentwerten ermöglicht in Abbildung 4 den direkten Vergleich mit Hungertieren und Tieren nach Proventrikel-Exstirpation.

Die durch eine Proventrikel-Exstirpation sonst zuverlässig herbeigeführten Effekte unterbleiben bei unmittelbar nachfolgender Reimplantation des entnommenen Organs: (1) In keinem Fall kam es zu permanenter Stolonisation; sofern überhaupt Fortpflanzungsprozesse auftraten, handelte es sich immer nur um einen einmaligen Vorgang. (2) In $70 \%$ der Fälle (36 Tiere) konnten weitere Fortpflanzungsvorgänge sogar vollständig unterbunden werden. 15 Exemplare $(30 \%)$ gelangten trotz der Reimplantation noch einmal zu Stolonisation: 2 von 27 vormaligen $q$ 우 (je 1 unter Bildung eines $\delta$ bzw. q Stolons) und 13 von $24 \delta \delta$ (stets wieder unter Bildung von $\delta$ Stolonen). 5 weitere $\delta \delta$ 
traten zwar zunächst in die geschlechtliche Reifung ein, resorbierten aber nach wenigen Tagen ihre Gonien wieder und kamen nicht zur Teilung. (3) Sofern trotz der Reimplantation eine Stolonisation auftrat, erfolgte die Teilung signifikant später als im Fall alleiniger Exstirpation. Erste Hinweise auf einen beginnenden StolonisationsprozeB zeigten sich zwar im Mittel bereits um den 8. Tag herum (d. h. nur 1,7 Tage später als im Fall der Exstirpation ohne nachfolgende Reimplantation), die weitere geschlechtliche Entwicklung vollzog sich jedoch deutlich langsamer (Abb. 5): Der Zeitraum zwischen sichtbarem Eintritt in eine Fortpflanzungsperiode und der Teilung betrug nach einer Proventrikel-Exstirpation im Mittel 8,0 Tage, nach einer Exstirpation mit Reimplantation aber 12,9 Tage und näherte sich damit dem entsprechenden Wert für intakte Tiere (14,4 Tage).

12 Tieren, die sich seit der Operation nicht mehr fortgepflanzt hatten, wurde der implantierte Proventrikel zwischen dem 70. und 75. Tag wieder entnommen. Alle Exemplare gelangten daraufhin innerhalb von 19 Tagen zur Fortpflanzung.

Der Ausgang dieser Versuchsreihe beweist die Möglichkeit einer Ersatztherapie der Exstirpationseffekte durch eine Proventrikel-Implantation. Damit ist sichergestellt, daß das Entfernen einer im bzw. am Proventrikel lokalisierten Hormonquelle und keine sonstwie geartete Beeinträchtigung des Versuchsobjektes durch die Operation für die Effekte von Proventrikel-Exstirpationen verantwortlich ist. Nach vollzogener Teilung wird der frühzeitige Eintritt in eine weitere Fortpflanzungsperiode und ein damit verbundener Ausfall der Regenerationsperiode unter dem Einfluß eines ProventrikelInkrets verhindert.

Exstirpierte und anschließend implantierte Proventrikel waren aber zunächst nicht immer in der Lage, die genau gleiche inhibitorische Aktivität zu entfalten, die sie ohne Operation unter sonst vergleichbaren Bedingungen (Hungerzustand) in situ ausgeübt hätten. Eine der Exstirpation unmittelbar folgende Proventrikel-Reimplantation konnte zwar in der Mehrzahl der Fälle weitere Fortpflanzungsvorgänge verhindern, jedoch gelangten die Versuchstiere signifikant häufiger und auch zu einem im Mittel früheren Termin zur Stolonisation als intakte Hungertiere (vergl. Abb. 4a und 4c). Erst nach Ablauf einer gewissen Zeit vermochten implantierte Proventrikel das Auftreten weiterer Fortpflanzungsprozesse genauso zuverlässig zu verhindern wie in situ belassene Proventrikel intakter Hungertiere. Eine mögliche Ursache dieses reinen Operationseffektes ist die durch den operativen Eingriff herbeigeführte vaskuläre Isolation des Proventrikels: Im Fall einer bevorzugten Verbreitung über das Blutgefäßsystem wäre die Abgabe des Proventrikel-Inkrets nach einer Operation zwangsläufig erschwert; der abfallende Inhibitorspiegel könnte dann die Prozesse der sexuellen Reifung und Stolonisation in Gang setzen, bis im Zuge der Ausbildung neuer Gefäßverbindungen die Hemmung wieder ihre volle Wirksamkeit erlangte.

\section{Nachweis einer rhythmischen endokrinen Aktivität von Proventrikeln im Transplantationsexperiment}

Das endokrine System des Proventrikels ist nach den dargestellten Ergebnissen zumindest insofern an der Kontrolle des Fortpflanzungsrhythmus beteiligt, als unter seinem Einfluß der unmittelbare Ubergang von einer Fortpflanzungsperiode in die nächste verhindert und eine zwischenzeitliche Regeneration ermöglicht wird. Noch 
ungeklärt ist, welche Faktoren das Ende der Regenerationsperiode und den Eintritt in eine neue Periode der geschlechtlichen Entwicklung bestimmen. Mit zunehmendem Abstand vom letzten Fortpflanzungstermin und fortschreitender Regeneration könnten innere Bedingungen auftreten, die schließlich eine weitere Hemmung der Stolonisationsprozesse durch das Proventrikel-Inkret unterbinden, obgleich dieses qualitativ und quantitativ unverändert vorliegt. Andererseits könnte gerade eine rhythmische Verminderung der inhibitorischen Aktivität des Proventrikels die periodische Induktion von sexueller Reifung und epitoker Metamorphose bewirken. Hierbei wäre zunächst von untergeordneter Bedeutung, ob der rhythmische Aktivitätsabfall eine autonome oder eine abhängige Leistung des Proventrikels darstellt. Mit der Transplantationsmethode wurden Proventrikel auf mögliche rhythmische Änderungen ihrer hormonalen Aktivität hin untersucht.

Den Empfängertieren wurde an einem Tag, an dem sie sich unter Abschnürung eines Stolons geteilt hatten, der Proventrikel entfernt und durch den eines Spendertieres ersetzt. Alle Empfänger befanden sich folglich zum Zeitpunkt des operativen Eingriffs im gleichen Stadium des Fortpflanzungszyklus. Die Empfänger der Gruppe A erhielten Proventrikel aus Spendern implantiert, die sich ebenfalls kurz zuvor geteilt hatten (am selben Tag wie die Empfänger oder am Tag zuvor), also der gleichen Zyklusphase wie die Empfänger entstammten. Die Empfänger der Gruppe B erhielten hingegen Proventrikel aus Spendern, die gerade äußerlich erkennbar in eine Stolonisationsperiode eingetreten waren, die sie 13-15 Tage später mit einer Teilung abgeschlossen hätten. Die Spender der Gruppe B befanden sich somit in einem Stadium des Fortpflanzungszyklus, das gegenüber demjenigen der Spender von Gruppe A (sowie gegenüber demjenigen der Empfänger) um etwa die halbe Periodenlänge phasenverschoben war. Als Empfänger dienten ausschlieBlich ơ $\delta$, als Spender Tiere beiderlei Geschlechts.

Die Ergebnisse der bereits behandelten Reimplantationsexperimente konnten auch für den Fall von Proventrikel-Transplantationen bestätigt werden: Tiere, die im Anschluß an eine Exstirpation wieder einen Proventrikel implantiert bekommen (sei es der eigene oder der eines anderen Individuums), verhalten sich nach einer gewissen Zeit wie intakte Hungertiere. Sofern überhaupt noch Fortpflanzungsprozesse auftreten, geschieht dies innerhalb der ersten 4 Wochen nach der Operation. Danach vermögen implantierte Proventrikel Stolonisationsvorgänge zuverlässig zu verhindern; niemals kommt es zu einer mehrmaligen Stolonisation.

Die Empfänger-Gruppen A und B zeigen signifikante Unterschiede sowohl hinsichtlich des Prozentsatzes stolonisierender Individuen als auch bezüglich des mittleren Teilungstermins (Abb. 6). Nur 35\% (28 von 81) der Versuchstiere der Gruppe A (mittlerer Teilungstermin 23,5 Tage nach der Operation), aber $83 \%$ (59 von 81) derjenigen von Gruppe B (mittlerer Teilungstermin 17,0 Tage nach der Operation) gelangten zur Stolonisation. 15 weitere Exemplare der Gruppe A und 2 der Gruppe B begannen zwar zunächst mit der Gametogenese, resorbierten jedoch die Gonien wieder und kamen nicht zur Teilung.

Einzig veränderter Parameter und somit Ursache des signifikant voneinander abweichenden Verhaltens der Empfängergruppen A und B ist die unterschiedliche Herkunft der transplantierten Proventrikel. Die von transplantierten Proventrikeln auf die Fortpflanzungsprozesse proventrikelloser Empfänger ausgeübte Hemmung erweist sich als unterschiedlich stark in Abhängigkeit zum Zustand des Spenders bei der 


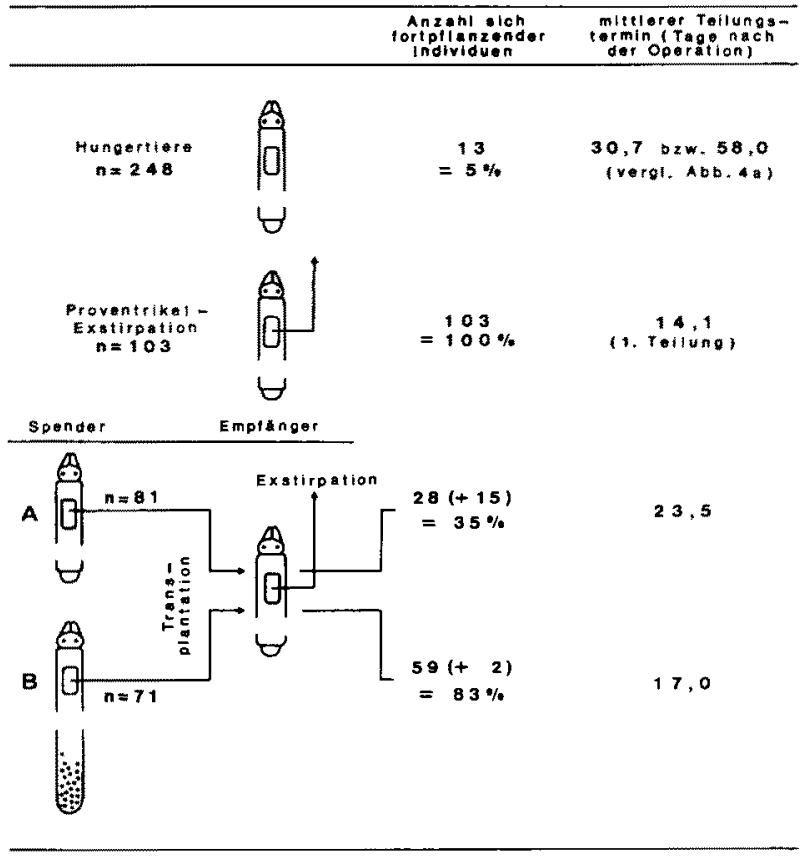

Abb. 6. Fortpflanzungsprozesse von Empfăngertieren nach einem direkt im Anschluß an eine Teilung vorgenommenen Ersatz des eigenen Proventrikels durch den von Spendertieren der Phase A des Fortpflanzungszyklus (gerade vollzogene Tellung) bzw. Phase B (gerade erfolgter Eintritt in eine Stolonisationsperiode); zum Vergleich: Tiere, die sich vom Tag einer Teilung an im Hungerzustand befanden, und Tiere, denen am Tag einer Teilung selektiv der Proventrikel entfernt worden ist $_{;}$in Klammern: Anzahl der Individuen, die zwar zunächst mit der Gametogenese begannen, aber nicht zur Teilung gelangten

Organübertragung: Proventrikel aus Spendern, die kurz zuvor eine Teilung vollzogen haben, entfalten als Implantat eine stärkere Inhibitorwirkung als solche aus Spendern, die gerade in eine Periode der geschlechtlichen Entwicklung eingetreten sind. Der Ausgang der Transplantationsversuche beweist somit die Existenz zyklischer Änderungen der endokrinen inhibitorischen Aktivität von Proventrikeln im Rhythmus des Wechsels von Regeneration und Fortpflanzungsvorgängen, d.h. mit einer spontanen mittleren Periodenlänge von etwa 30 Tagen. Dem Fortpflanzungsrhythmus liegt offensichtlich eine rhythmische endokrine Funktion des Proventrikels als unmittelbare Ursache zugrunde.

Die Tatsache, daß Empfänger der Gruppe A signifikant häufiger ( $35 \%$ gegenüber $5 \%$ ) und auch zu einem früheren mittleren Termin zur Fortpflanzung gelangten als intakte Hungertiere, ist Ausdruck des bereits erwähnten Operationseffektes. Hinsichtlich des Prozentsatzes stolonisierender Individuen und des mittleren Teilungstermins ist das Verhalten der Empfänger der Gruppe A demjenigen der Versuchstiere der Reimplantationsexperimente (Abb. 4c) vergleichbar. Der Effekt von ProventrikelImplantationen ist folglich unabhängig davon, ob es sich bei den Implantaten um die zuvor exstirpierten eigenen Organe oder die anderer Individuen aus gleicher Zyklusphase handelt. 
Überraschend ist der Befund, daß nicht sämtliche Tiere der Empfänger-Gruppe B zur Fortpflanzung gelangten, obgleich die implantierten Proventrikel aus Spendern stammten, die gerade in eine Stolonisationsperiode eingetreten waren, und obgleich der Operationseffekt das Auftreten von Fortpflanzungsprozessen zusätzlich gefördert haben muß. Die Ursache dieser unerwartet hohen inhibitorischen Aktivität von Proventrikeln aus Spendern der Phase B ist unbekannt. Verschiedene Erklärungen bieten sich an: Die Produktion und Sekretion von Hormonen unterliegt meist einer fördernden oder/und hemmenden Kontrolle durch übergeordnete Zentren sowie Einflüssen der Erfolgsorgane. Bei einer Transplantation endokriner Organe in Systeme abweichender innerer Bedingungen (wie im Fall der Proventrikel der Empfänger-Gruppe B) sind daher Änderungen der hormonalen Aktivität gegenüber den In-situ-Verhältnissen zu erwarten. Möglicherweise wird im vorliegenden Fall die Periode der geschlechtlichen Entwicklung zwar durch den Abfall der inhibitorischen Aktivität des Proventrikels induziert, eine für den Fortgang der sexuellen Reifung aber notwendige, anhaltende Aktivitätsverminderung jedoch erst über eine positive Rückkopplung seitens der heranreifenden Geschlechtsprodukte erreicht. Wird der Proventrikel diesem Einfluß durch eine Exstirpation entzogen, könnte seine endokrine Aktivität frühzeitig zu höheren Werten zurückkehren. Eine entsprechende Überlegung gilt auch für den Fall, daß die endokrine Funktion des Proventrikels ihrerseits einer Kontrolle z.B. durch das Gehirn unterliegen sollte: Eine Transplantation in Empfängertiere abweichender Zyklusphase würde das endokrine System des Proventrikels zwangsläufig veränderten Eỉnflüssen des ihm übergeordneten Steuerzentrums aussetzen.

\section{Der Einfluß exogener Faktoren auf die zeitliche Verteilung von Fortpflanzungsereignissen}

Die individuelle Fortpflanzungsaktivität ist ein endogen-rhythmischer ProzeB; seine unmittelbare Ursache sind wahrscheinlich periodische Änderungen der hormonalen Aktivität des Proventrikels. Verschiedene äußere Faktoren nehmen jedoch Einfluß auf das Fortpflanzungsgeschehen, indem sie entweder das Auftreten von Fortpflanzungsprozessen überhaupt verhindern oder aber modifizierend auf den Fortpflanzungstermin einwirken. Es liegt die Vermutung nahe, daß diese Faktoren ihre Wirkung letztlich über eine Einflußnahme auf die endokrine Funktion des Proventrikels ausüben.

\section{Verlust caudaler Segmente}

Der Verlust einer größeren Anzahl caudaler Segmente, wie er durch eine Amputation experimentell herbeigeführt werden kann, aber auch natürlicherweise häufig auftritt (hervorgerufen durch die Angriffe von Freßfeinden oder infolge Zerbrechens der fragilen Tiere beim Umherkriechen zwischen den Algenthalli ihres Habitats), sind weitere Fortpflanzungsprozesse und damit zusätzliche Segmentverluste zunächst unterbunden. Diese können jedoch jederzeit durch eine Proventrikel-Exstirpation induziert werden. Eine quantitative Untersuchung zur Beeinflussung des Fortpflanzungstermins durch Amputation caudaler Segmente liegt noch nicht vor. 


\section{Nahrungsentzug}

Der Einfluß von Hungerbedingungen auf die Fortpflanzungsaktivität wurde bereits behandelt: Tiere, die unmittelbar im Anschluß an eine Teilung in den Hungerzustand versetzt werden, zeigen i.d.R. keine weiteren Stolonisationen, die wegen des Ausfalls der Regeneration einen fortschreitenden Substanzverlust mit sich brächten. Die Körperreserven werden statt dessen zur Überdauerung der Hungerperiode verwendet. Eine Proventrikel-Exstirpation kann die Fortpflanzung jedoch jederzeit, auch nach einer 3 Monate währenden Hungerperiode, zuverlässig herbeiführen. Somit scheint weder das Fehlen eines Regenerats noch der Nährstoffmangel selbst die direkte Ursache der auftretenden Hemmung zu sein. Die einfachste Erklärung findet das Ausbleiben weiterer Fortpflanzungsprozesse in der Annahme, daß die periodische Verminderung der endokrinen Aktivität des Proventrikels im Hungerzustand unterbleibt bzw. nicht mehr das für die Induktion einer Stolonisation notwendige Ausmaß erreicht.

\section{Temperatur und Photoperiode}

4 Massenkulturen, in denen je etwa 50 Individuen unter konstanten Bedingungen $\left(20^{\circ} \mathrm{C}\right.$, LD 16:8) zur Geschlechtsreife aufgezogen worden waren, wurden von der 18. Woche an unterschiedlichen Kombinationen von Temperatur $\left(20^{\circ} \mathrm{C}, 12^{\circ} \mathrm{C}\right)$ und Photoperiode (LD 16:8, 10:14) ausgesetzt. Nach einer Anpassungszeit von 35 Tagen wurden die Kulturen über einen Zeitraum von 50 Tagen hin täglich nach abgeschnürten Stolonen durchsucht. Das Ergebnis zeigt Tabelle 1.

Tab. 1. Fortpflanzungsaktivität in Massenkulturen bei verschiedenen Kombinationen von Temperatur und Photoperiode. A: Anzahl der Fortpflanzungsprozesse während der 50tägigen Versuchsdauer bezogen auf den Mittelwert der Versuchstier-Anzahlen bei Beginn und Ende des Versuchs

\begin{tabular}{|c|c|c|c|c|c|}
\hline $\begin{array}{r}\text { Kultur } \\
\text { Temperatur }\end{array}$ & $\begin{array}{l}\text { dingungen } \\
\text { Photoperiode }\end{array}$ & \multicolumn{2}{|c|}{$\begin{array}{l}\text { Versuchstier-Anzahl } \\
\text { bei Versuchs- }\end{array}$} & $\begin{array}{c}\text { Anzahl der } \\
\text { Teilungsprozesse } \\
\text { (N) }\end{array}$ & $\begin{array}{l}\text { Fortpflanzungs- } \\
\text { aktivität (A) } \\
A=2 N /\left(n_{1}+n_{2}\right)\end{array}$ \\
\hline $20^{\circ} \mathrm{C}$ & $\begin{array}{l}\text { LD 16: } 8 \\
\text { LD 10:14 }\end{array}$ & $\begin{array}{l}52 \\
50\end{array}$ & $\begin{array}{l}41 \\
42\end{array}$ & $\begin{array}{l}71 \\
41\end{array}$ & $\begin{array}{l}1,53 \\
0,89\end{array}$ \\
\hline $12^{\circ} \mathrm{C}$ & $\begin{array}{l}\text { LD 16: } 8 \\
\text { LD 10:14 }\end{array}$ & $\begin{array}{l}49 \\
51\end{array}$ & $\begin{array}{l}42 \\
49\end{array}$ & $\begin{array}{r}33 \\
1\end{array}$ & $\begin{array}{l}0,68 \\
0,02\end{array}$ \\
\hline
\end{tabular}

Temperatur und Photoperiode haben einen deutlichen Einfluß auf die Fortpflanzungsaktivität. Eine Kombination von Kurztagbedingungen (LD 10:14) und tiefer Temperatur $\left(12^{\circ} \mathrm{C}\right)$ vermag Fortpflanzungsprozesse nahezu vollständig zu unterbinden, wozu weder der eine noch der andere Faktor allein in der Lage ist. Der langsam fortschreitende Wachstumsprozeß wird von keinen Segmentverlusten infolge weiterer Stolonisationen mehr unterbrochen. Tiere, die für längere Zeit unter diesen Bedingungen gehalten werden, wachsen folglich allmählich zu Individuen von beträchtlicher Körpergröße heran (Länge im narkotisierten Zustand bis 30 mm, PpS-Anzahl bis 95), wie sie im Freiland nur selten, und dann auch nur im Frühjahr, anzutreffen sind. 


\section{Synchronisation der individuellen Fortpflanzungsrhythmen durch ein periodisches Belichtungsprogramm}

Eine Freilanduntersuchung läßt eine deutliche Tendenz innerhalb der Population zur Synchronisation der individuellen Fortpflanzungsrhythmen erkennen. Die zeitliche Verteilung von Fortpflanzungsprozessen zeigt ausgeprägte Maxima und Minima in Beziehung zum Mondphasenwechsel. Materialproben aus dem Habitat der untersuchten Population enthielten während der gesamten Fortpflanzungssaison stets auch eine Anzahl Tiere, die kurz vor einer Teilung standen; der relative Anteil solcher Exemplare war jedoch während der 1. Mondviertel jeweils beträchtlich größer (10-15mal) als zu anderen Zeiten. Auch ein Abfischen der oberflächennahen Wasserschicht über einem stark besiedelten Habitat kurz nach einsetzender Morgendämmerung lieferte deutliche Hinweise auf ein lunarperiodisch gehäuftes Auftreten reifer Stolonen im Freiland.

Die individuellen Fortpflanzungsrhythmen, und damit auch die ihnen sehr wahrscheinlich als unmittelbare Ursache zugrunde liegenden endokrinen Aktivitätszyklen der Proventrikel, müssen folglich in ihrer Phasenlage durch einen exogenen Faktor beeinflußbar sein, der als Zeitgeber die endogenen Rhythmen der Individuen einer Population mit einer äußeren Periodik, nämlich dem Mondphasenwechsel, synchronisiert. Wahrscheinlicher Zeitgeber des lunaren Fortpflanzungsrhythmus ist das Mondlicht, d.h. die mit den Mondphasen wechselnde Nachthelligkeit; dies wird durch den Ausgang des folgenden Laborexperiments nahegelegt.

Als Versuchsmaterial dienten Massenkultur-Tiere (10 Massenkulturen mit je 40-60 Individuen), die unter konstanten Bedingungen (20 ${ }^{\circ} \mathrm{C}, \mathrm{LD}$ 16:8) aufgezogen worden waren und im Alter von 11-13 Wochen ihre erste Stolonisation durchgeführt hatten. Eine anfängliche Tendenz zur Synchronisation, die sich aus der Gleichaltrigkeit der Versuchstiere erklärt, verlor sich zwischen dem 150. und 210. Tag; die Fortpflanzungsprozesse in den Massenkulturen erfolgten nun annähernd gleichmäßig über die Zeit verteilt (Abb. 7a). Am 210. Tag wurden die Tiere zu gleichen Teilen zwei verschiedenen Versuchsgruppen zugeordnet, die beide einem identischen, aber um die halbe Perio-

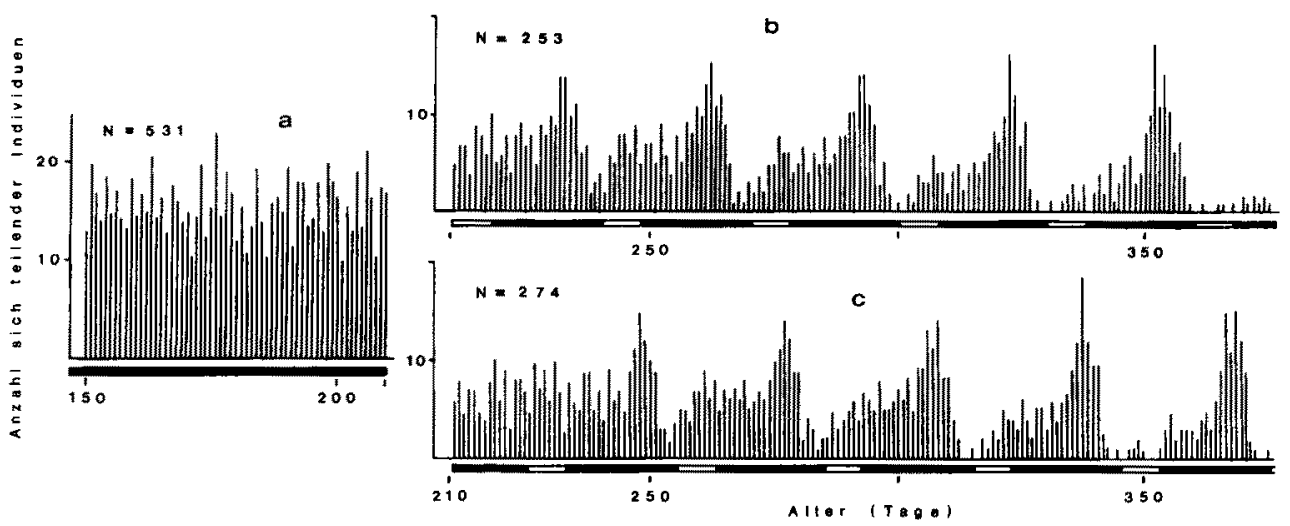

$\mathrm{Abb}$. 7. Zeitliche Verteilung von Fortpflanzungsprozessen gleichaltriger Massenkultur-Tiere unter konstanten Bedingungen (bis zum Alter von 210 Tagen) (a) und unter dem EinfluB eines periodischen Belichtungsprogramms (b, $c)$; $\mathbf{a r}$ : Phasen mit dunklen Nächten; $\square \square$ : Phasen mit hellen

Nächten. Weitere Erläuterungen im Text 
denlänge phasenverschobenen rhythmischen Belichtungsprogramm ausgesetzt wurden. In einem 30tägigen Zyklus wechselte jeweils eine 22tägige Periode (1.-22. Zyklustag) mit dunklen Nächten (täglich 16 Std. Licht einer 15-Watt-Lampe und 8 Std. absolute Dunkelheit) und eine 8tägige Periode (23.-30. Zyklustag) mit hellen Nächten (Dauerlicht LL einer 15-Watt-Lampe).

Abbildung $7 \mathrm{~b}$, $\mathrm{c}$ zeigt den synchronisierenden Effekt, den ein solches periodisches Belichtungsprogramm auf das Fortpflanzungsgeschehen in Massenkulturen auszuüben vermag. Die Teilungsmaxima lagen jeweils zu Beginn der zweiten Hälfte einer Dunkelphase und entstanden deutlich erkennbar auf Kosten ihnen unmittelbar folgender Minima. Individuen, die sich im Fall konstanter Bedingungen zu Zeiten der Minimumphasen geteilt hätten, befanden sich folglich während der LL-Perioden des Belichtungszyklus in einer sensiblen Phase, in der sie durch einen mit dem Dauerlicht-Impuls verbundenen, aber noch unbekannten Parameter zu einem etwas vorzeitigen Eintritt in die geschlechtlichen Entwicklungsprozesse veranlaßt werden konnten.

\section{DISKUSSION}

Die Untersuchungen an Typosyllis prolifera zeigen die herausragende Bedeutung eines im bzw. am Proventrikel lokalisierten endokrinen Organs oder Gewebes für die Kontrolle der zeitlichen Verteilung von Fortpflanzungsprozessen.

Zyklische Anderungen der endokrinen Aktivität dieses Systems mit einer mittleren spontanen Periodenlänge von etwa 30 Tagen sind die unmittelbare Ursache eines endogenen Fortpflanzungsrhythmus. Im Anschluß an eine Teilung durchläuft der endokrine Aktivitätszyklus seine Maximumphase. Der hohe Hormontiter verhindert den unmittelbaren Übergang in eine weitere Fortpflanzungsperiode und ermöglicht eine zwischenzeitliche Regeneration der zuvor als Stolon verlorengegangenen Segmente. Das Zusammenspiel von Regeneration und Stolonisation garantiert eine annähernde Konstanz des mittleren Segmentumfanges eines Tieres über die aufeinanderfolgenden Fortpflanzungszyklen hinweg. Eine direkte Hormonabhängigkeit der Regeneration konnte bisher nicht nachgewiesen werden; das Proventrikel-Inkret fördert den Regenerationsvorgang vermutlich nur indirekt über die Hemmung der antagonistischen geschlechtlichen Entwicklung. Mit dem Abfall der endokrinen Aktivität des Proventrikels erfolgt der Wechsel von der Regenerations- zur neuen Stolonisationsperiode: Die Proliferation und Differenzierung der Gonien werden induziert; parallel zur sexuellen Reifung, jedoch prinzipiell unabhängig von ihr, vollziehen sich die Prozesse der stolonialen Metamorphose; die caudale Segmentneubildung kommt zum Stillstand.

Periodische Änderungen der endokrinen Aktivität von Proventrikeln im Rhythmus des Wechsels von Regenerations- und Fortpflanzungsprozessen sind in Transplantationsexperimenten nachgewiesen. Der genaue zeitliche Verlauf und das relative Ausmaß der Anderungen des Hormontiters sind jedoch unbekannt; ihre Ermittlung durch Injektionsexperimente in einem biologischen Testverfahren ist wegen der geringen Körpergröße der Versuchstiere kaum realisierbar.

Exogene Faktoren nehmen in verschiedener Weise Einfluß auf die zeitliche Verteilung von Fortpflanzungsereignissen,wobei sie vermutlich an der endokrinen Funktion des Proventrikels angreifen. Bestimmte Verhältnisse hinsichtlich Temperatur und Photoperiode bilden Voraussetzungen für das Auftreten von Fortpflanzungsprozessen. 
Kurztagbedingungen und niedere Wassertemperaturen hemmen die Stolonisation und sind vermutlich diejenigen Umweltfaktoren, die im Freiland während der Wintermonate eine Unterbrechung der Fortpflanzungstätigkeit zugunsten einer längeren Wachstumsperiode erzwingen. Ein Einfluß der Photoperiode auf Stolonisationsprozesse ist kürzlich auch von einem anderen Sylliden, Autolytus prolifer, bekanntgeworden (Schiedges, 1977).

Während einer ausgedehnten Fortpflanzungssaison sind im Freiland alle Voraussetzungen für den Ablauf von Stolonisationsprozessen erfüllt. Adulte Individuen kommen während dieses Zeitraums offensichtlich wiederholt zur Fortpflanzung, entsprechend dem unter Laborbedingungen gefundenen endogenen Fortpflanzungsrhythmus. Die Fortpflanzungsaktivität erfährt aber immer auch eine Anpassung an die individuellen Lebensumstände: Hungerbedingungen sowie der Verlust einer größeren Anzahl caudaler Segmente führen zu einem (vorübergehenden) Ausfall weiterer Fortpflanzungsprozesse, die unter diesen Umständen zu einer drastischen Verringerung der Segmentanzahl führen würden. Insbesondere der Verlust caudaler Segmente infolge Zerbrechens der fragilen Tiere ist eine häufige natürliche Erscheinung.

Eine weitere Funktion exogener Faktoren besteht in der Synchronisation von Fortpflanzungsvorgängen innerhalb einer Population. Lunare Periodizitätsphänomene sind bei Polychaeten weit verbreitet (Korringa, 1947; Olive \& Clark,1978). An Laborkulturen des lunarrhythmisch schwärmenden Nereiden Platynereis dumerilii konnte Hauenschild $(1956,1960,1961)$ erstmals den experimentellen Beweis für eine photoperiodische Steuerung einer Lunarperiodizität erbringen. Die Verkürzung der wirksamen Photoperiode bei abnehmendem Mond erwies sich als derjenige Faktor, der - offensichtlich über eine Drosselung der cerebralen Hormonsekretion - bei allen reaktionsfähigen Individuen einer Population synchron die Endphase der geschlechtlichen Entwicklung in Gang setzt und damit das synchrone Schwärmen programmiert. Das Mondlicht wird hierbei möglicherweise ñicht unmittelbar auslösend wirksam, sondern fungiert als synchronisierender Zeitgeber eines endogenen, lunaren Rhythmus der Metamorphosebereitschaft. Während die Schwärmperiodizitäten der monotelischen Nereiden ein reines Populationsphänomen darstellen, ist das lunarrhythmisch gehäufte Auftreten reifer Stolonen von Typosyllis prolifera im Freiland das Ergebnis einer Synchronisation individueller Fortpflanzungsrhythmen. Auch in diesem Fall scheint das Mondlicht die Rolle des Zeitgebers zu spielen, der die endogenen Fortpflanzungsrhythmen (bzw. die diesen zugrunde liegenden hormonalen Rhythmen) der Individuen einer Population mit dem Mondphasenwechsel synchronisiert. Ein künstliches periodisches Belichtungsprogramm, das in stark vereinfachter Form den 30tägigen Mondlichtzyklus simuliert, kann im Labor eine Synchronisation der unter konstanten Bedingungen freilaufenden Rhythmen herbeiführen. Der wirksame Parameter des Mondphasenwechsels ist bisher unbekannt.

Bei fast allen in dieser Hinsicht experimentell untersuchten Polychaeten konnte das Prostomium als Zentrum einer hormonalen Kontrolle der Fortpflanzungserscheinungen identifiziert werden. Neurosekretorische Zellen des Gehirns gelten als Urheber der endokrinen Funktionen des Prostomiums. Die Sylliden bilden eine bemerkenswerte Ausnahme: Der für die Steuerung von Fortpflanzungsvorgängen unmittelbar verantwortliche endokrine Faktor entstammt nicht dem Prostomium, sondern einer davon räumlich deutlich getrennten Struktur der Pharynxregion. Bis heute fehlt eine histo- 
physiologische Bestätigung der allein aus experimentellen Untersuchungen erschlossenen endokrinen Funktionen der Pharynxregion. Golding (1972) äußert die Vermutung, daß Elemente des stomatogastrischen Nervensystems die gesuchten Hormonproduzenten darstellen könnten. Aber auch ein nicht dem Nervensystem zugehöriges Gewebe könnte für die beschriebenen hormonalen Effekte veantwortlich sein, wenngleich ein solcher Fall von Anneliden bisher unbekannt ist. Möglicherweise vermag eine parallele Untersuchung von Syllinen und Autolytinen zur histologischen Identifizierung des endokrinen Systems beizutragen: Aus den experimentellen Untersuchungen ist bekannt, daß dieses System zwar in beiden Fällen in der Pharynxregion lokalisiert ist, aber nur bei Syllinen eine derart enge Verbindung mit dem Proventrikel eingeht, daß es mit diesem zusammen exstirpiert und transplantiert werden kann.

Das endokrine System des Proventrikels erscheint nach den Ergebnissen der vorgelegten Untersuchung als Zentrum umfangreicher regulatorischer Funktionen. Es stellt sich jedoch die Frage, ob diese Funktionen tatsächlich in autonomer Regie ausgeübt werden, oder ob die endokrine Aktivität von Proventrikeln nicht möglicherweise ihrerseits einer Kontrolle seitens des Gehirns unterliegt, das den Einfluß von Außenbedingungen auf die Stolonisation vermittelt und eventuell sogar selbst primärer Sitz der endogenen Rhythmik ist. Erste Versuchsergebnisse deuten in der Tat darauf hin, daß das endokrine System der Proventrikelregion einer ebenfalls endokrinen Steuerung durch das Prostomium unterliegt. Hiermit wird erstmals für einen Anneliden die Existenz einer Hormonhierarchie angedeutet. Dieses Problem ist Gegenstand weiterer Untersuchungen.

\section{ZITIERTE LITERATUR}

Abeloos, M., 1950. Régénération et stolonisation épigame chez l'Annélide Syllis prolifera Krohn. C. r. hebd. Séanc. Acad. Sci., Paris (D) 230, 1899-1900.

Durchon, M., 1950. Maturation génitale précoce, stolonisation et polycéphalie, par suppression de la région antérieure chez un Syllidien. - C. r. hebd. Séanc. Acad. Sci., Paris (D) 230, 2236-2237.

Durchon, M., 1951. Stolonisation et hermaphrodisme successif chez Syllis amica. - Archs Zool. exp. gén. 88, 96-100.

Durchon, M., 1952. Recherches expérimentales sur deux aspects de la reproduction chez les Annélides polychètes: L'épitoquie et la stolonisation. - Annls Sci. nat. (Zool. Biol, anim.) 14, 117-206.

Durchon, M., 1957. Rôle du proventricule dans le déterminisme de la stolonisation chez les Syllidiens. - C. r. hebd. Séanc. Acad. Sci, Paris (D) 244, 1283-1286.

Durchon, M., 1959. Contribution à l'étude de la stolonisation chez les Syllidiens; I. Syllinae. - Bull. biol. Fr. Belg. 93, 155-219.

Durchon. M., 1970. Déterminisme endocrine de la maturation sexuelle chez les Annelides polychètes. - Bull. Soc. zool. Fr, 95, 489-509.

Durchon, M., 1975. Sex reversal in the Syllinae. In: Intersexuality in the animal kingdom. Ed. by R. Reinboth. Springer, Berlin, 41-47.

Durchon. M. \& Wissocq, J.-C., 1964. Contribution à l'étude de la stolonisation chez les Syllidiens: II. Autolytinae. - Annls Sci. nat. (Zool. Biol. anim.) 6, 159-208.

Franke, H.-D., 1977. Die Rolle des Proventrikels bei der Steuerung von Fortpflanzungsrhythmus und sexueller Differenzierung des Polychaeten Typosyllis prolifera (Syllidae). - Verh. dt. zool. Ges. 70, 324 .

Golding, D. W., 1972. Studies in the comparative neuroendocrinology of polychaete reproduction.Gen. comp. Endocrinol. (Suppl.) 3, 580-590.

Hauenschild, C., 1956. Neue experimentelle Untersuchungen zum Problem der Lunarperiodizität. Naturwissenschaften $43,361-363$. 
Hauenschild, C., 1959. Hemmender Einfluß der Proventrikelregion auf Stolonisation und Oocytenentwicklung bei dem Polychaeten Autolytus prolifer. - Z. Naturf, 14b, 87-89.

Hauenschild, C., 1960. Lunar periodicity. - Cold Spring Harb. Symp. quant. Biol. 25, 491-497.

Hauenschild, C., 1961. Die Schwärmperiodizität von Platynereis dumerilii im DD/LL-Belichtungszyklus und nach Augenausschaltung. $-\mathrm{Z}$. Naturf. 16b, 753-756.

Hauenschild, C., 1974. Endokrine Beeinflussung der geschlechtlichen Entwicklung einiger Polychaeten. - Fortschr. Zool. 22 (2/3), 75-92.

Hauenschild, C., 1975. Die Beteiligung endokriner Mechanismen an der geschlechtlichen Entwicklung und Fortpflanzung von Polychaeten. - Verh. dt. zool. Ges. 67, 292-308.

Junqua, C., 1957. Stolonisation et polycéphalie expérimentales chez Trypanosyllis zebra (Annélide Polychète). -- Annls Sci. nat. (Zool. Biol. anim.) 19, 59-68.

Korringa, P., 1947. Relation between the moon und periodicity in the breeding of marine animals. Ecol. Monogr. 17, 347-381.

Malaquin, A., 1893. Recherches sur les Syllidiens. Morphologie, anatomie, reproduction, développement. - Mém. Soc. Sci. Agric. Lille 4 (18), 1-477.

Okada, Yo K., 1929. Regeneration and fragmentation in the syllidian polychaetes. - Wilhelm Roux Arch. EntwMech. Org. 115, 542-600.

Olive, P. J. W. \& Clark, R. B., 1978. Physiology of reproduction. In: Physiology of annelids. Ed. by P. J. Mill. Acad. Press, London, 271-368.

Potts, F. A., 1911. Methods of reproduction in the Syllids. - Ergebn. Fortschr. Zool. 3, 1-72.

Schiedges, K.-L., 1977. Entwicklungsphysiologische Untersuchungen zur Fortpflanzung, Entwicklung und Regeneration sowie deren Steuerung bei den Polychaeten (Sylliden) Autolytus prolifer und Autolytus brachycephalus. Diss., Univ. Köln, 146 pp.

Schroeder, P. C. \& Hermans, C. O., 1975. Annelida, Polychaeta. In: Reproduction of marine invertebrates. Ed. by A. C. Giese \& J. S. Pearse. Acad. Press, New York, 3, 1-213.

Wissocq, J.-C., 1963-1964. Nouveaux cas d'inversion sexuelle chez les Syllidiens. - Mém. Soc. natn. Sci. nat. math. Cherbourg 51, 105-109.

Wissocq, J.-C., 1966. Rôle du proventricule dans le déterminisme de la stolonisation chez Syllis amica. - C. r. hebd. Séanc. Acad. Sci., Paris (D) 262, 2605-2608. 